\title{
Isolated congenital left ventricular diverticulum presenting as stable angina pectoris and surgical treatment
}

\author{
Vusal Veliyev ${ }^{1}$, Hikmet Sahratov², Tamara Musayeva ${ }^{1}$ \\ ${ }^{1}$ Department of Cardiology, Azerbaijan Military Hospital, Baku, Azerbaijan \\ ${ }^{2}$ Department of Cardiovascular Surgery, Azerbaijan Military Hospital, Baku, Azerbaijan
}

Kardiochirurgia i Torakochirugia Polska 2019; 16 (1): 44-46

A congenital left ventricular diverticulum (LVD) is a relatively rare cardiac anomaly and has to be differentiated from an aneurysm or pseudoaneurysm. A recent large single-center study demonstrated that the prevalence of the left ventricular diverticulum in adults is $0.42 \%$ and one third of the affected patients had nonfatal cardiovascular events during follow-up [1]. Major concerns are thrombosis, embolism, rupture, congestive heart failure, ventricular arrhythmias and sudden cardiac death. In adults, LVD can be misdiagnosed as other types of heart diseases, especially when the present symptom is angina pectoris. In this report, we describe a congenital LVD on the apical wall presenting stable angina pectoris in an adult patient. The patient did not respond to conservative treatment and underwent successful surgical treatment.

A 43-year-old man was referred to our clinic because of complaints of angina-like chest pain, mainly on heavy exertion. The patient described the pain as tightness located in the retrosternal area with no radiation. The chest pain typically lasted 3 to 5 minutes and ordinary physical activity did not cause angina. His only risk factor was excess weight. The patient had no significant previous medical history.

On physical examination, no abnormal findings were obtained. The electrocardiogram showed $\mathrm{T}$ wave flattening/inversion on inferior leads. It was not suggestive of ischemia. The results of laboratory tests were unremarkable and also cardiac biomarkers were negative. A chest $X$-ray showed no abnormality and there was no clue about the source of the chest pain.

Two-dimensional transthoracic echocardiography (TTE), from the modified apical four-chamber view demonstrated "bird beak"-like outpouching on the apex of the left ventricle. During systole the volume of the outpouching was decreasing. The TTE ruled out associated congenital anomalies (Fig. 1).

We interpreted that the outpouching was probably an aneurysm, as a result of coronary artery disease. The patient underwent conventional coronary angiography which demonstrated mild coronary artery disease. A subsequent left ventriculography, obtained in $30^{\circ}$ right anterior oblique projection, showed "bird beak"-like outpouching on the apical wall (Fig. 2).

Contrast-enhanced cardiac magnetic resonance imaging (MRI) with its advanced sequences characterized this outpouching very well and helped us to understand it was a congenital LVD (Fig. 3).

The patient, who underwent medical treatment, had no improvement in his complaints after one-month follow-up and surgical treatment decision was made. Median sternotomy was performed and after opening the pericardium, a bird beak-like diverticulum structure was observed in the left ventricular apex (Fig. 4). Pledget-supported purse string sutures were placed along the narrow-necked diverticulum circumference at the beating heart. The sutures were stretched, and transesophageal echocardiography con-

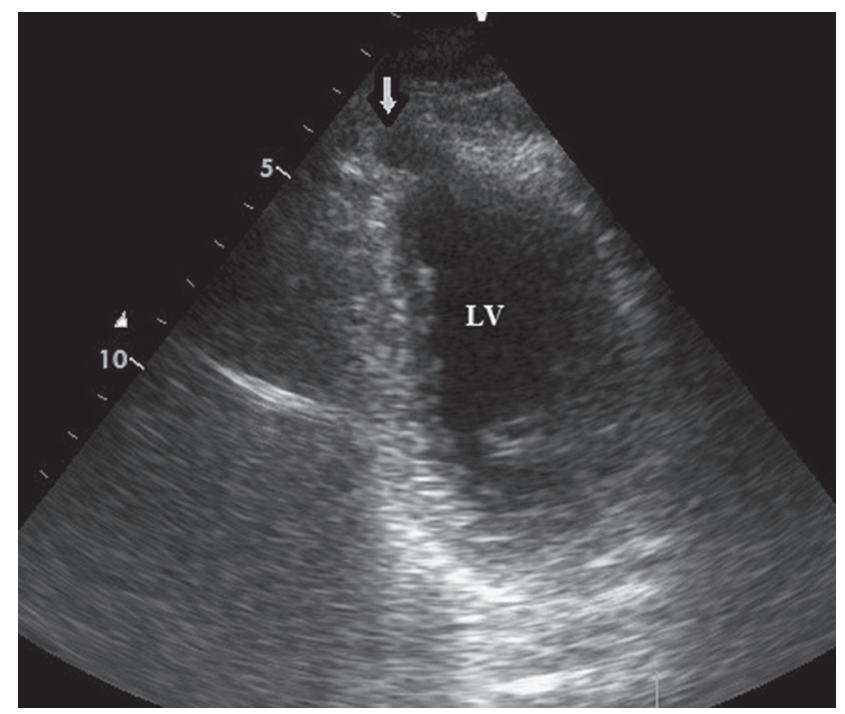

Fig. 1. 2D transthoracic echocardiography, modified apical views, showed outpouching on the apical wall during diastole (arrow) LV - left ventricle.

Address for correspondence: Hikmet Sahratov MD, PhD, Department of Cardiovascular Surgery, Azerbaijan Military Hospital, Baku, Azerbaijan, phone: +99 4504821213, e-mail: sahratzade@gmail.com

Received: 31.10.2018, accepted: 13.12.2018. 


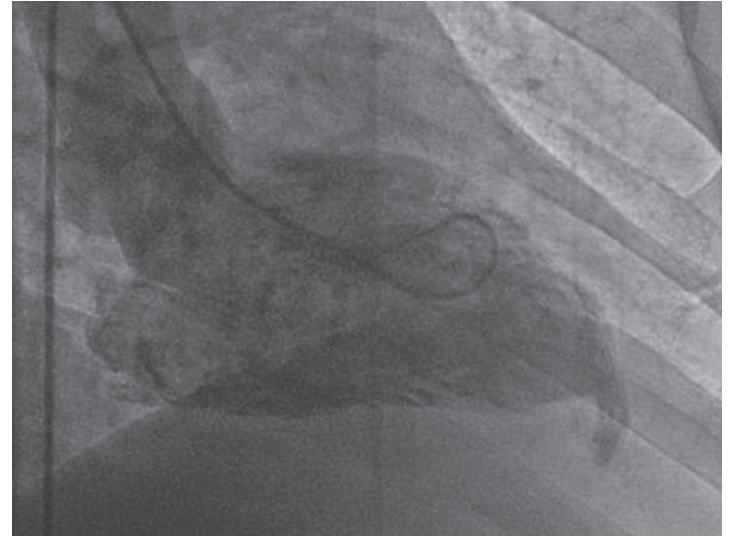

Fig. 2. Left ventriculography in right anterior oblique view illustrates the diverticulum originating from the apical wall of the left ventricle with contraction during systole

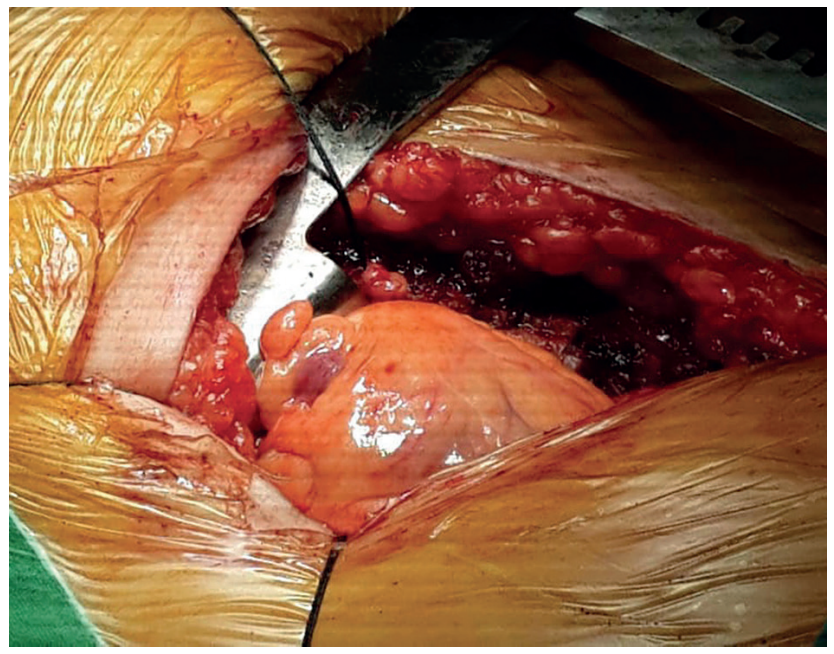

Fig. 4. The appearance of the diverticulum in the left ventricle apex after opening the pericardium

firmed the disappearance of the diverticulum connection with the left ventricular cavity. After the supportive sutures were placed, the operation was terminated (Fig. 5). In the early postoperative period, the patient reported no symptoms of angina. The postoperative period was uneventful, and the patient was discharged after 5 days. In the postoperative $1^{\text {st }}$ month and $1^{\text {st }}$ year control examinations, the patient did not have anginal symptoms.

Congenital LVD is a relatively rare cardiac anomaly. A large single-center, retrospective study suggests that the prevalence of LVD in adults is $0.42 \%$ [1]. Other cardiac, vascular, or thoracoabdominal abnormalities place together in approximately $70 \%$ of patients and the other $30 \%$ of patients are isolated, such as in our case report. The LVD may be congenital or acquired, the former being more common. These are considered to be congenital if there is no history of conditions that have injured the myocardium (for example, infection, traumatic, surgery, or tumor).

Possible etiologies of the congenital LVD include intrinsic abnormalities during embryogenesis, such as mal-

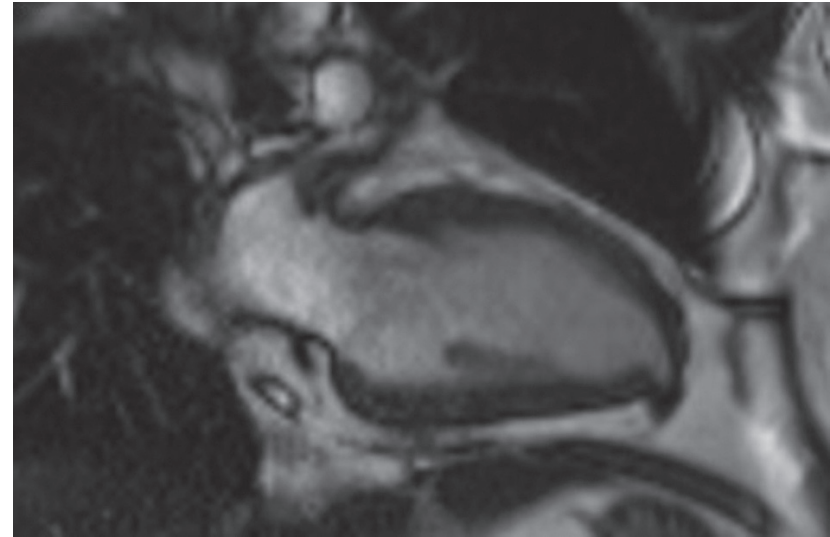

Fig. 3. Cardiac magnetic resonance imaging vertical long-axis (2-chamber) gradient echo sequences demonstrate a congenital left ventricular diverticulum. In the diastole, the diverticulum was $11 \times 6 \mathrm{~mm}$ in size

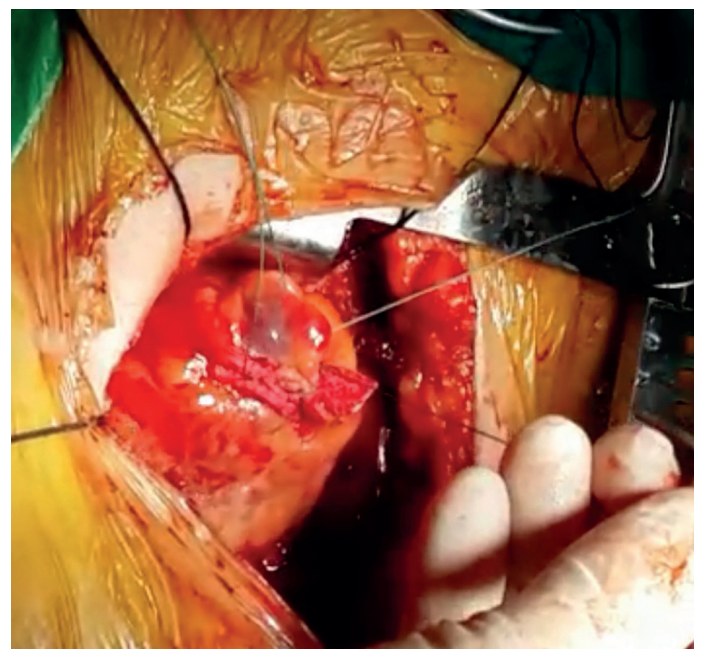

Fig. 5. Diverticulum after insertion of purse string suture with pledgets

development of myocardial intraventricular sinusoids or acquired malformations in utero, including coronary artery abnormalities, viral infections, or arrhythmia related vascular accidents, which may result in a localized weakness of the LV wall with gradual protrusion, due to the high intraventricular pressure [2].

Histologically, LVD are classified into fibrous and muscular types. The former is prone to rupture.

When such LVD are detected, the main differential diagnoses are pseudoaneurysm, and less frequently takotsubo cardiomyopathy and myocardial crypts. Certain methods can help to differentiate them. Muscular LVD can contract synchronously with the rest of the left ventricle and is associated with normal ejection fraction. Normal coronary arteries favor LVD. The varied shapes of the anatomy (fingerlike, hook-like, conical, lobulated, cylindrical shapes) and visualizing smaller anatomical sizes support the LVD. A diverticulum includes all layers of the heart (endocardium, myocardium, and pericardium). A diverticulum has a wide or narrow neck communicating with the ventricle. Differen- 
tiation is important because of varying management options, potential complications and prognosis.

Although no conclusive or gold standard diagnostic methods have been established, echocardiography, heart catheterization, computed tomography, and MRI are useful diagnostic tools. Cardiac MRI is emerging as a very useful tool that allows simultaneous anatomical and functional evaluation along with tissue characterization, which has diagnostic, therapeutic and prognostic implications [3].

Most patients are asymptomatic, but others may complain of chest pain, chest distress, palpitation and exertional dyspnea. Clinical presentation can vary; myocardial infarction, transient ischemic attacks, congestive heart failure, valve regurgitation, supraventricular tachycardia, ventricular tachycardia, ventricular wall rupture, and sudden cardiac death have been reported in the literature [4].

The treatment options of a congenital left diverticulum are still undefined. There are no randomized trials for analysis of treatment regimens, and data have been derived from case reports. Surgical correction may be considered under some circumstances, including: 1) progressive thinning of the wall of the LVD, 2) right ventricular diverticulum, 3) surgery is required for other reasons, 4) large diverticulum or increasing size over time, 5) development of heart failure, 6) occurrence of complications, 7) systolic dyskinesia or paradoxical bulging, 8) if medical therapy is ineffective, etc. $[5,6]$. Surgical treatment of asymptomatic cases is still controversial. Cardiovascular disease (CVD) has the potential to cause complications such as embolism, arrhythmia, heart failure and rupture. Some authors have recommended resection of all CVDs to avoid these complications, while others have recommended conservative treatment $[7,8]$. Since our case was symptomatic and the patient had chest pain with medical treatment, we decided to perform surgical treatment.

The LVD is rare but may be associated with high morbidity and mortality rates. Differential diagnosis of the LVD from other left ventricular outpouchings is important, because their prognosis varies widely from benign to catastrophic. There is scarce information available about its definition, natural history, management strategy, and treatment approaches in the literature. Surgical treatment should be considered as an option when necessary.

\section{Disclosure}

The authors report no conflict of interest.

\section{References}

1. Ohlow MA, Secknus MA, Geller JC, von Korn H, Lauer B. Prevalence and outcome of congenital left ventricular aneurysms and diverticula in an adult population. Cardiology 2009; 112: 287-293.

2. Brachlow A, Sable C, Smith S, Slack M, Martin G. Fetal diagnosis and postnatal follow-up of an asymptomatic congenital left ventricular diverticulum. Pediatr Cardiol 2002; 23: 658-660.

3. Sharma A, Kumar S. Overview of left ventricular outpouchings on cardiac magnetic resonance imaging. Cardiovasc Diagn Ther 2015; 5: 464-470.

4. Ohlow MA, von Korn H, Lauer B. Characteristics and outcome of congenital left ventricular aneurysm and diverticulum: analysis of 809 cases published since 1816. Int J Cardiol 2015; 185: 34-45.

5. Elshaer F, Altwijri F, Alrasheed F, Kharabsheh S, Habeeb W. Congenital ventricular diverticulum and $\mathrm{MI}$ - diagnostic challenges and implications. J Saudi Heart Assoc 2015; 27: 277-282.

6. Jung JC, Oh HC, Kim KH. Isolated congenital left ventricular diverticulum in adults. Korean J Thorac Cardiovasc Surg 2015; 48: 355-358.

7. Marijon E, Ou P, Fermont L, Concordet S, Le Bidois J, Sidi D, Bonnet D. Diagnosis and outcome in congenital ventricular diverticulum and aneurysm. J Thorac Cardiovasc Surg 2006; 131: 433-437.

8. Jain S, Mahajan R, Rohit MK. Percutaneous transcatheter device closure of an isolated congenital LV diverticulum: first case report. Pediatr Cardiol 2011; 32: $1219-1222$. 